\title{
Development and Evaluation of a New Interpenetrating Network Bead of Sodium Carboxymethyl Xanthan and Sodium Alginate for Ibuprofen Release
}

\author{
Rajat Ray, Siddhartha Maity, Sanchita Mandal, Tapan K. Chatterjee, Biswanath Sa \\ The Division of Pharmaceutics, Department of Pharmaceutical Technology, Jadavpur University, Kolkata, India. \\ Email: biswanathsa2003@yahoo.com
}

Received June $6^{\text {th }}, 2010$; accepted July $8^{\text {th }}, 2010$.

\begin{abstract}
Interpenetrating network (IPN) beads of sodium carboxymethyl xanthan (SCMX) and sodium alginate (SAL) were prepared by ionotropic gelation process using $\mathrm{AlCl}_{3}$ as a cross-linking agent. The effect of different formulation variables like total polymer concentration, gelation time, concentration of cross-linking agent, and drug load on the extent of release of ibuprofen (IBP), a non steroidal anti-inflammatory drug, was examined. The formation of IPN structure was examined using Fourier Transform Infra-red (FTIR) analysis and the compatibility of the drug in the bead was evaluated through FTIR, X-ray diffraction (XRD) and Differential Scanning Calorimetry (DSC) analyses. While increase in the concentration of total polymer, gelation time, and drug load decreased the drug release in both acidic ( $p H-1.2)$ and phosphate buffer $(P B)$ solution ( $p H-6.8)$, increase in the concentration of cross-linking agent tended to increase the drug release. However, from all the formulations, the drug release in acidic medium was considerably slow and a maximum 14\% of the loaded drug was released in 2 h. Complete drug release was achieved in PB solution within 210 to 330 min depending upon the formulation variables. The release of the drug followed non-Fickian transport process in acidic medium and case-II transport mechanism in PB solution and these release behaviour correlated well with the kinetics of dynamic swelling of IPN beads. The study indicated that the IPN beads of SCMX and SAL could be a suitable dosage form to minimize the drug release in acidic solution and to control the drug release in PB solution depending upon the need.
\end{abstract}

Keywords: IPN Bead, Ibuprofen, Drug Release, Kinetics, Swelling

\section{Introduction}

Among the most abundant natural polymers, polysaccharides are widely used in pharmaceutical dosage forms as excipients like suspending agents, emulsifying agents, tablet binders, gelling agents. With the advent of macromolecular chemistry, the use of polysaccharides has been extended towards new applications in pharmaceutical, biomedical, and agricultural fields. Although naturally available polysaccharides exhibit certain limitations in terms of their reactivity and processibility, these can be overcome by modification of the polysaccharides through either physical or chemical cross-linking, grafting with other materials and developing hydrogels or interpenetrating network(IPN) structures.

Since the homopolymers alone can not meet divergent demand in terms of properties and performances, development of IPN appears to be a better approach [1] and one of the easiest ways for modification of the properties of polysaccharides. IPN consists of two polymers, each in network form, which can be cross-linked in the presence of each other to give a three dimensional network structure [2] and hence, combine the properties of two cross-linked polymers in a network form [3]. IPNs are thus emerging as a rapidly developing branch of polymer blended technology and are finding applications in artificial implants, dialysis, membranes, drug delivery systems [4], and in agricultural field [5].

Sodium alginate (SAL), a hydrophilic biopolymer obtained from brown sea weeds, is a polysaccharide composed of varying proportions of D-mannuronic acid (M) and L-guluronic acid $(\mathrm{G})$ residues which are arranged in 
MM or GG blocks interspersed with MG blocks [6]. Its unique property of forming water insoluble calcium alginate gel through ionotropic gelation with $\mathrm{Ca}^{2+}$ ions in a simple and mild condition has made possible to encapsulate both macromolecular agents [7] and low molecular weight therapeutic agents $[8,9]$ in calcium alginate beads. However in physiological environment, calcium alginate beads tend to have poor mechanical stability [10]. To overcome this limitation, IPN beads of SAL with gelatin or egg albumin [2], polyvinyl alcohol-graftedpolyacrylamide [11], N,O-Carboxymethyl chitosan [12] for controlled drug delivery, and with gelatin [5] for controlled release of pesticides have been developed.

Although xanthan gum, a polysaccharide obtained from Xanthomonas campestris, can not form gel beads, its Na-salt of carboxymethyl derivative is able to form gel beads through ionotropic gelation with $\mathrm{Al}^{3+}$ ions [13]. Sodium carboxymethyl xanthan (SCMX) beads have been found capable of encapsulating albumin [14] and diltiazem hydrochloride [15]. However hitherto there are no reports on IPN beads of SCMX with SAL for drug release study.

The objective of the present work was to develop a new IPN bead composed of SCMX and SAL and to evaluate the beads for encapsulation and release behavior of ibuprofen (IBP).

\section{Experimental}

\subsection{Materials}

Ibuprofen (Indian Pharmacopoeia) and xanthan gum were obtained as gift samples from respectively $\mathrm{M} / \mathrm{S}$ Albert David Limited and M/S Deys Medical Stores (Mfg). Pvt. Limited, Kolkata, India. Sodium alginate (Mol. wt. 240kDa), $\mathrm{AlCl}_{3} \cdot 2 \mathrm{H}_{2} \mathrm{O}$ (SD Fine Chem Pvt. Ltd, Mumbai, India), Monochloro acetic acid (Loba Chemie Pvt. Ltd, Mumbai, India) and all other analytical grade reagents were obtained commercially and used as received.

\subsection{Preparation of Sodium Carboxymethyl Xanthan (SCMX)}

Xanthan gum was derivatised to SCMX having O-carboxymethyl substitution of 0.8 following the method reported previously [13]. In brief, required amount of xanthan gum was dispersed in ice cold solution of $45 \% \mathrm{w} / \mathrm{v}$ sodium hydroxide. The dispersion was kept at $5-8^{\circ} \mathrm{C}$ with continuous stirring for $1 \mathrm{~h}$. Monochloroacetic acid solution $(75 \% \mathrm{w} / \mathrm{v})$ was added with stirring in the reaction mixture and the temperature was raised slowly to $15-18^{\circ} \mathrm{C}$. After $30 \mathrm{~min}$, the temperature was raised to $75^{\circ} \mathrm{C}$ and maintained for additional $30 \mathrm{~min}$. The reaction mixture was, then cooled to room temperature, cut into small pieces and dried at $50^{\circ} \mathrm{C}$. The dried product was milled, washed with $80 \% \mathrm{v} / \mathrm{v}$ methanol and again dried.

\subsection{Preparation of Interpenetrating Network (IPN) Bead}

Required amount of ibuprofen (IBP) was homogenously dispersed in an aqueous solution of SCMX and SAL. The resulting dispersion was extruded through $21 \mathrm{G}$ flat-tip hypodermic needle into $\mathrm{AlCl}_{3}$ solution. Gelation of the beads was carried out for different periods of time. The beads were, then collected by filtration, washed with deionized water, dried at $45^{\circ} \mathrm{C}$ in a hot air oven to constant weight and kept in a dessicator until used. The beads were prepared using the following variables:

1) Keeping the drug load constant at $50 \% \mathrm{w} / \mathrm{w}$ of total polymer and the concentration of $\mathrm{AlCl}_{3}$ constant at $2 \%$ $\mathrm{w} / \mathrm{v}$, the total polymer concentration was varied from $2-4 \% \mathrm{w} / \mathrm{v}$ (SCMX to SAL weight ratio 1:1) and the gelation time was varied from 0.5 to 2 hour.

2) Keeping the drug load constant at $50 \% \mathrm{w} / \mathrm{w}$ of total polymer, the gelation time at 0.5 hour and total polymer concentration $3 \% \mathrm{w} / \mathrm{v}$, concentration of $\mathrm{AlCl}_{3}$ was varied from $2-8 \% \mathrm{w} / \mathrm{v}$.

3) Keeping the total polymer concentration fixed at $3 \% \mathrm{w} / \mathrm{v}$, gelation time at 0.5 hour, and $\mathrm{AlCl}_{3}$ concentration at $2 \% \mathrm{w} / \mathrm{v}$, drug load was varied from $20-60 \% \mathrm{w} / \mathrm{w}$ of total polymer. The composition of beads is shown in Table 1. Each formulation was prepared in duplicate.

Table 1. Composition and drug entrapment efficiency (DEE) of sodium carboxymethyl xanthan (SCMX) and sodium alginate (SAL) IPN beads

\begin{tabular}{ccccc}
\hline $\begin{array}{c}\text { SCMX\%: } \\
\text { SAL\% }\end{array}$ & $\begin{array}{c}\text { Drug load } \\
\text { total polymer })\end{array}$ & $\begin{array}{c}\text { Gelation } \\
\text { time }(\mathrm{hr})\end{array}$ & $\begin{array}{c}\text { Concentration } \\
\text { of AlCl3 } \\
(\% \mathrm{w} / \mathrm{v})\end{array}$ & $\begin{array}{c}\mathrm{DEE} \\
(\mathrm{Mean} \pm \mathrm{SD}, \\
\mathrm{n}=4)\end{array}$ \\
\hline $1: 1$ & 50 & 0.5 & 2 & $93.46 \pm 2.18$ \\
$1.5: 1.5$ & 50 & 0.5 & 2 & $97.22 \pm 2.45$ \\
$2: 2$ & 50 & 0.5 & 2 & $99.50 \pm 2.86$ \\
$1: 1$ & 50 & 2 & 2 & $91.15 \pm 1.92$ \\
$1.5: 1.5$ & 50 & 2 & 2 & $94.25 \pm 3.37$ \\
$2: 2$ & 50 & 2 & 2 & $95.31 \pm 2.72$ \\
$1.5: 1.5$ & 50 & 0.5 & 4 & $97.25 \pm 1.38$ \\
$1.5: 1.5$ & 50 & 0.5 & 8 & $97.65 \pm 3.84$ \\
$1.5: 1.5$ & 20 & 0.5 & 2 & $98.86 \pm 1.26$ \\
$1.5: 1.5$ & 40 & 0.5 & 2 & $99.16 \pm 1.74$ \\
$1.5: 1.5$ & 60 & 0.5 & 2 & $96.96 \pm 2.52$ \\
\hline
\end{tabular}




\subsection{Fourier Transform Infrared (FTIR) Analysis}

FTIR spectra of SCMX, SAL and drug free IPN bead were recorded in a FTIR spectrophotometer (Perkin- Elmer, model Spectrum RX-1, UK). Each sample was mixed with $\mathrm{KBr}$ and converted into disc at $100 \mathrm{~kg}$ pressure using a hydraulic press. The spectra were recorded within $4000-400 \mathrm{~cm}^{-1}$ wave numbers. Similarly, the FTIR spectra of IBP and drug loaded IPN beads were recorded.

\subsection{Powder X-Ray Diffraction (XRD) Analysis}

Qualitative XRD studies were performed using an X-ray diffractometer (Bruker $\mathrm{D}_{8}$ advanced powder diffractometer, USA). Pure IBP and powdered beads were scanned from $5^{\circ}$ to $55^{\circ}$ diffraction angle $(2 \theta)$ range under the following conditions:

Source, Ni-filtered $\mathrm{Cu}-\mathrm{K} \alpha(\lambda=1.54)$ radiation; voltage, $40 \mathrm{kV}$; Current, $40 \mathrm{~mA}$; scan speed, $16^{\circ} / \mathrm{min}$

\subsection{Differential Scanning Calorimetry (DSC) Study}

DSC thermograms of IBP and powdered beads were obtained in the following way:

A weighed amount (about $6 \mathrm{mg}$ ) of sample was kept in a hermetically sealed aluminium pan and heated at a scan speed of $10^{\circ} \mathrm{C} / \mathrm{min}$ over a temperature range of $35^{\circ} \mathrm{C}$ $310^{\circ} \mathrm{C}$ in a Differential Scanning Calorimeter (PerkinElmer, model Pyris Diamond TG/DTA, UK ) which was calibrated against indium. A nitrogen purge $(20 \mathrm{ml} / \mathrm{min})$ was used throughout the runs.

\subsection{Photomicrograph}

Photomicrograph of IPN beads were taken at $4 \mathrm{X}$ magnification with an optical microscope (Leica DM 2500P) fitted with a camera (Cannon Power Shot S-80, Japan).

\subsection{Drug Entrapment Efficiency}

IPN beads $(20 \mathrm{mg})$ were accurately weighed in an electronic balance (Precisa XB $600 \mathrm{MC}$, Precisa Instrument Ltd; Switzerland), immersed in $250 \mathrm{ml}$ USP phosphate buffer (PB) solution ( $\mathrm{pH}$ 6.8), and shaken for $2 \mathrm{~h}$ on a mechanical shaker. The beads were crushed and further shaken for $1 \mathrm{~h}$. The solution was filtered and an aliquot following suitable dilution was analyzed at $222 \mathrm{~nm}$ in a UV-Visible spectrophotometer (model Cary-50 Bio-spectrophotometer, VARIAN, Australia)) and the content of the beads was determined using a calibration curve constructed using PB solution of $\mathrm{pH}$ 6.8. The reliability of the above analytical method was judged by conducting recovery analysis at three levels of spiked drug solution in the presence or absence of the polymers for three consecutive days. The recovery averaged $98.45 \pm 2.68 \%$. DEE was determined using the following relation:

DEE $(\%)=($ Determined drug content/Theoretical drug content) $\times 100$

\subsection{In-Vitro Drug Release Study}

In-vitro drug release study was carried out in acidic solution $0.1(\mathrm{~N}) \mathrm{HCl}(\mathrm{pH} \mathrm{1.2)}$ and in USP PB solution $(\mathrm{pH}$ 6.8) using USP-II dissolution rate test apparatus (model TDP-06P Electro Lab, Mumbai, India). $20 \mathrm{mg}$ beads were placed in $500 \mathrm{ml}$ acidic solution or $500 \mathrm{ml} \mathrm{PB}$ solution $\left(37 \pm 1{ }^{\circ} \mathrm{C}\right)$ and rotated with paddle at $75 \mathrm{rpm}$. Aliquot was withdrawn at different times and replenished immediately with the same volume of fresh solution. Undiluted or suitably diluted withdrawn samples were analyzed spectrophotometrically at $220 \mathrm{~nm}$ for acidic solution and $222 \mathrm{~nm}$ for PB solution. The amount of drug released in acidic solution and PB solution were calculated from the calibration curves drawn respectively, in $0.1(\mathrm{~N}) \mathrm{HCl}$ and $\mathrm{PB}$ solution ( $\mathrm{pH}$ 6.8). Each release study was conducted four times.

\subsection{Swelling Study}

Dried drug-free IPN beads $(50 \mathrm{mg})$ were immersed in $25 \mathrm{ml}$ acidic solution $(\mathrm{pH} 1.2)$ at $37^{\circ} \mathrm{C}$. The beads were removed at different times by filtration and blotted carefully to remove excess surface water. The swollen beads were weighed. The swelling ratio of the beads were determined using the following formula:

Swelling ratio $=$ (weight of swollen beads-weight of dry beads)/weight of dry beads

Swelling ratio of the beads in $\mathrm{PB}$ solution $(\mathrm{pH}$ 6.8) was determined in a similar way.

\subsection{Statistical Analysis}

Each formulation was prepared in duplicate, and each analysis was duplicated. Effect of formulation variables on drug release was tested for significance level by using analysis of variance (ANOVA: single factor and two factor) with the aid of Microsoft ${ }^{\circledR}$ Excel 2003. Difference was considered significant when $\mathrm{p}<0.05$.

\section{Results \& Discussion}

\subsection{Formation of IPN}

IPN beads composed of SCMX and SAL were prepared by inotropic gelation process using $\mathrm{AlCl}_{3}$ as a common cross-linking agent for both the polymers. Formulation of IPN structure was verified by FTIR analysis (Figure 1). FTIR spectrum of SCMX showed the presence of bands corresponding to asymmetric and symmetric carboxylate anions at respectively $1605 \mathrm{~cm}^{-1}$ and $1419 \mathrm{~cm}^{-1}$, a broad band at $3419 \mathrm{~cm}^{-1}$ corresponding to stretching vibration of hydroxyl group, a peak at $1327 \mathrm{~cm}^{-1}$ corresponding to $\mathrm{C}=\mathrm{O}$ stretching of carboxymethyl group. These results are similar to the findings reported earlier [14]. The spectrum of SAL showed the bands characteristics of 


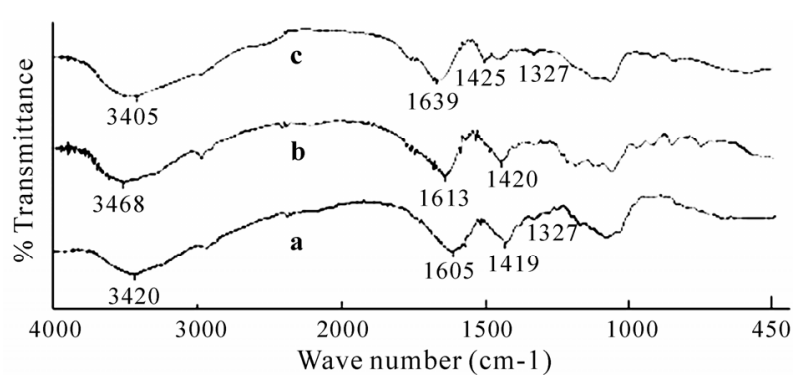

Figure 1. FTIR spectra of (a) SCMX, (b) SAL, (c) drug free IPN bead

asymmetric and symmetric carboxylate anions at respectively $1612 \mathrm{~cm}^{-1}$ and $1420 \mathrm{~cm}^{-1}$, a broad peak corresponding to the stretching of hydroxyl group at 3468 $\mathrm{cm}^{-1}$. Similar spectrum of SAL has been reported elsewhere [16]. The FTIR spectrum of drug-free IPN beads showed peaks at $1639 \mathrm{~cm}^{-1}$ and $1425 \mathrm{~cm}^{-1}$ respectively for asymmetric and symmetric carboxylate anions and a peak at $3405 \mathrm{~cm}^{-1}$ for hydroxyl group. Moreover, the peak at $1327 \mathrm{~cm}^{-1}$ assigned for carboxymethyl group was retained. Comparison of the spectra, however, demonstrated shift of the peaks of carboxylate anions to higher wave numbers. The shift of carboxylate bands confirms the formation of complex between the two polymers and $\mathrm{Al}^{3+}$ ions through physical cross-linking. These results suggest the formation of IPN structure wherein both the polymers are present in cross-linked condition.

\subsection{Morphology of IPN Bead}

The composition of IBP-loaded IPN beads has been shown in Table1. The beads were prepared with a SCMX to SAL weight ratio of $1: 1$ but in different total polymer concentration $(1 \%: 1 \%, 1.5 \%: 1.5 \%, 2 \%: 2 \%)$ and gelling in $\mathrm{AlCl}_{3}$ solution $(2-8 \% \mathrm{w} / \mathrm{v})$ for different periods of time $(0.5$ to $2 \mathrm{~h})$. Although the shapes of the wet beads were spherical, the shapes distorted after drying. The surface of dried beads was rough and folded (Figure 2) and was due to shrinkage of the beads during the drying process. Similar shape distortion has been reported for chitosan/

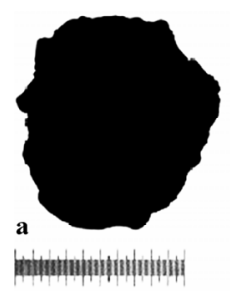

$0.01 \mathrm{~mm}$

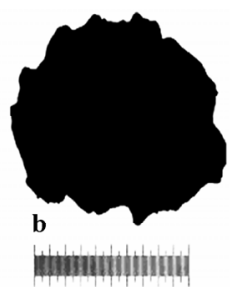

$0.01 \mathrm{~mm}$

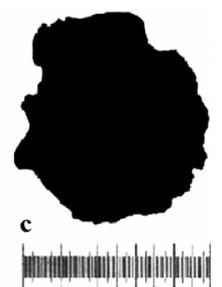

$0.01 \mathrm{~mm}$
Figure 2. Photo micrographs of ibuprofen-loaded IPN beads, prepared under different conditions. (a) SCMX: SAL 1\%:1\%, 2\% w/v $\mathrm{AlCl}_{3}, 0.5 \mathrm{~h}$, (b) SCMX: SAL 1.5\%: $1.5 \%, 4 \% \mathrm{w} / \mathrm{v} \mathrm{AlCl}_{3}, 2 \mathrm{~h}$, (c) SCMX: SAL 2\%:2\%, 8\% w/v $\mathrm{AlCl}_{3}, 0.5 \mathrm{~h}$ carageenan beads [17]. Moreover, neither the concentration of cross-linking agent $\left(\mathrm{AlCl}_{3}\right)$ nor the gelation time had any appreciable effect on morphology of IPN beads. The shape of the beads did not change even when the drug load was varied from 20 to $60 \% \mathrm{w} / \mathrm{w}$ of total polymer.

\subsection{Compatibility of Drug in IPN Bead}

Compatibility of IBP in IPN beads was studied using FTIR, XRD and DSC analyses. The characteristics bands corresponding to $\mathrm{C}=\mathrm{O}$ stretching and $-\mathrm{OH}$ stretching of IBP appeared in FTIR spectrum respectively at $1720 \mathrm{~cm}^{-1}$ and $2956 \mathrm{~cm}^{-1}$. The above two bands were also detected at the same positions in the spectrum of drug-loaded IPN beads (Figure 3). XRD analysis showed reflection to the interplanner distances of $14.41,7.24,5.32,5.01,4.72$, $4.65,4.39,3.98$ and $3.63 \AA$ respectively at $6.13,12.21$, $16.64,17.68,18.78,19.06,20.20,22.30$ and $24.52^{\circ} 2 \theta$. Drug-loaded IPN beads also exhibited the same reflections at the same $2 \theta$ degrees (Figure 4). The result indicates that the crystallinity of the drug in IPN beads was retained and no amorphization of the drug took place. Comparison of DSC thermograms revealed that the

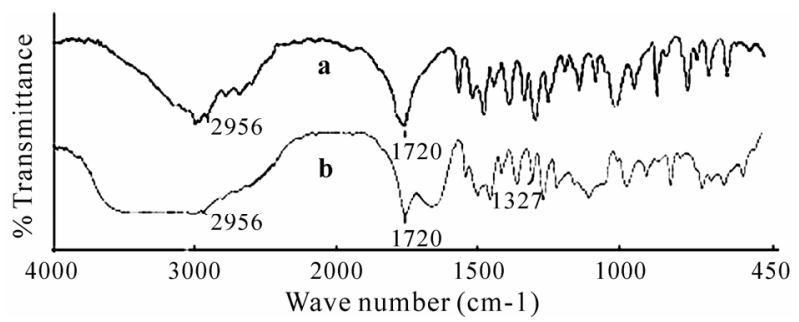

Figure 3. FTIR spectra of (a) Ibuprofen (b) Ibuprofen-loaded IPN bead

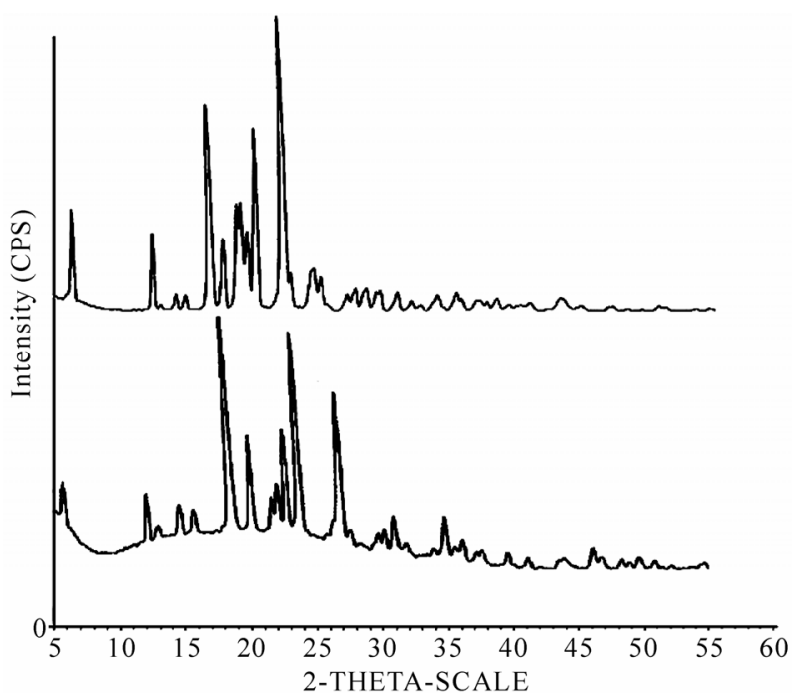

Figure 4. X-ray diffractograms of (a) Ibuprofen (b) Ibuprofen-loaded IPN bead 
melting endothermic peak of IBP at $79^{\circ} \mathrm{C}$ also appeared in the DSC curve of drug loaded IPN bead (Figure 5). These studies indicated that apparently no interactions between the drug and the polymers took place during the formation of IPN beads.

\subsection{DEE of IPN bead}

DEE of IPN beads tended to increase as the total polymer concentrations was increased from $2-4 \% \mathrm{w} / \mathrm{w}$ keeping SCMX to SAL weight ratio constant at 1:1 (Table 1). Two way analysis of variance (ANOVA-2) revealed significant difference $\left(\mathrm{F}_{2,3}>\mathrm{F}_{\text {tabular }}\right.$ at 0.05 level $)$ in DEE in IPN beads prepared with increasing polymer concentrations although no significant difference was noted within the batches of each formulation. Same observations were noted with IPN beads which were prepared by gelling in $0.5 \% \mathrm{AlCl}_{3}$ solution for two different gelation times. Increase in DEE with increase in total polymer concentrations is related to the higher rigidity of the matrices of IPN beads. Higher encapsulation efficiency of cefadroxyl has been reported for IPN beads prepared using SAL and gelatin or egg albumin [2]. Concentrations of cross-linking agent did not produce any appreciable change in DEE of IPN beads prepared with a total polymer concentration of $3 \% \mathrm{w} / \mathrm{v}$ keeping SCMX to SAL weight ratio constant at 1:1. The results of one way analysis of variance (ANOVA-1) revealed no significant difference in DEE ( $\mathrm{F}_{2,3}<\mathrm{F}_{\text {tabular }}$ at 0.95 level) of IPN beads prepared with various concentrations of $\mathrm{AlCl}_{3}$. Similar independence of DEE on the extent of cross-linking has been reported for ketorolac loaded IPN beads composed of sodium carboxymethyl cellulose and gelatin [18]. The time of gelation, however, had an impact on DEE which tended to decrease as gelation time was increased (Table 1). The results are in agreement with the reports of other workers [2]. Although the solubility of IBP in aqueous medium is very less, prolonged exposure in the gelation medium may cause greater leaching of the drug from IPN beads resulting in decreased DEE. IPN beads having 20 to $60 \% \mathrm{w} / \mathrm{w}$ of IBP were prepared using $3 \% \mathrm{w} / \mathrm{w}$ total polymer concentration and gelling for $0.5 \mathrm{~h}$ in $2 \% \mathrm{w} / \mathrm{v}$ $\mathrm{AlCl}_{3}$ solution. DEE was found to vary within 96.96 to $99.16 \%$ (Table 1). No significant effect of drug loading on DEE was observed. Similar non-dependence of DEE on $\%$ of drug loading has been reported for IPN beads composed of sodium carboxymethyl cellulose and gelatin.

\subsection{In-Vitro Drug Release}

\subsubsection{Effect of Polymer Concentration}

Release of IBP from IPN beads, prepared using increased polymer concentrations (SCMX: SAL weight ratio 1:1) and gelling for $0.5 \mathrm{~h}$ in $2 \% \mathrm{AlCl}_{3}$ solution, have been represented in Figure 6. Drug release in acidic medium

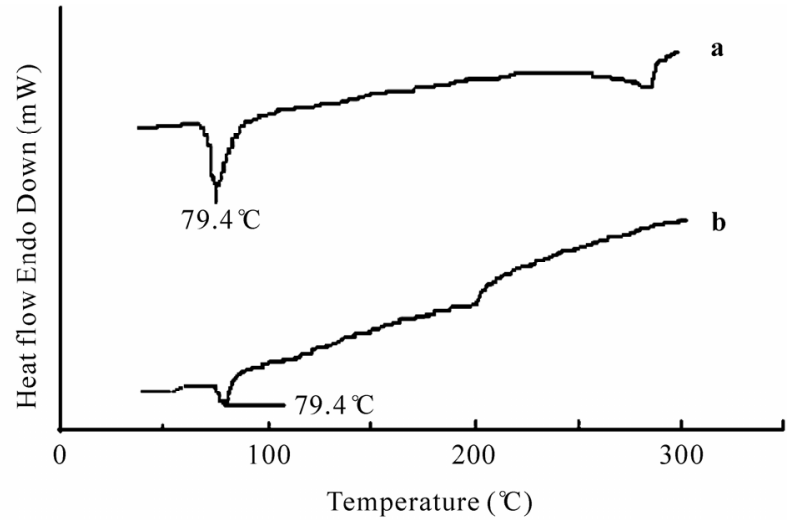

Figure 5. DSC thermograms of (a) Ibuprofen (b) Ibuprofen-loaded IPN bead

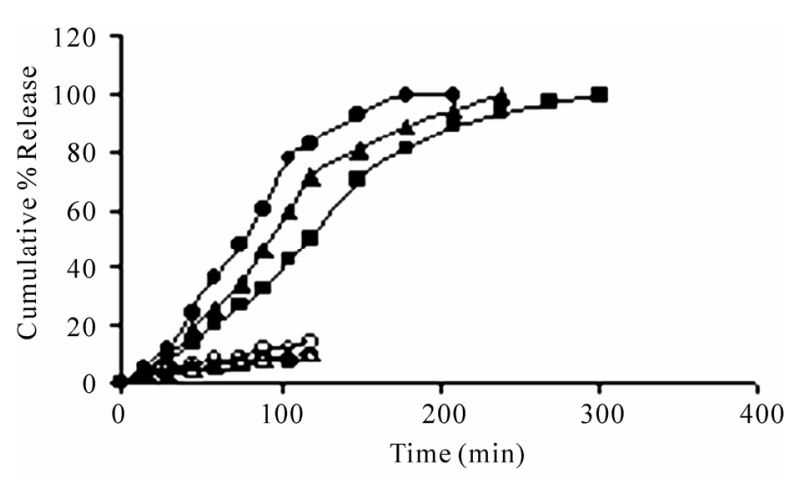

Figure 6. Release profiles of Ibuprofen in acidic solution (open symbols) and phosphate buffer solution (closed symbols) from IPN beads prepared using different concentration of SCMX and SAL and gelling in $2 \% \mathrm{w} / \mathrm{v} \mathrm{AlCl}_{3}$ solution for $0.5 \mathrm{~h}$. Key: SCMX: SAL $=(O)$ 1\%:1\%, $(\Delta)$ 1.5\%:1.5\%, ( $\square$ ) 2\%:2\%. Maximum SEM = 1.24(n=4)

was slow and 8.82 to $14.09 \%$ of the loaded drug was released in $2 \mathrm{~h}$. In PB solution ( $\mathrm{pH}$ 6.8), complete drug release was achieved in $210 \mathrm{~min}$ to $300 \mathrm{~min}$ depending upon the total polymer concentration in the beads. Increase in total polymer concentration from 2 to $4 \% \mathrm{w} / \mathrm{w}$ decrease the drug release in both the dissolution media. The derived properties obtained from drug release profiles indicated that the area under the curves (AUCs), determined using trapezoidal rule, in acidic dissolution medium decreased as the polymer concentration in IPN beads increased. Similarly, the time required for $50 \%$ $\left(\mathrm{t}_{50 \%}\right)$ and $80 \%\left(\mathrm{t}_{80 \%}\right)$ drug release in PB solution increased and AUCs decreased with increase in polymer concentration in the beads. Similar trend in drug release was observed from IPN beads which were prepared by gelling for $2 \mathrm{~h}$ (Table 2). Drug release from hydrophilic polymeric beads depends upon the type of matrix used as well as its rigidity [11]. Increase in total polymer concentration results in a more entangled or more compact gel system with a greater cross-linking density in the 
Table 2. Derived properties of drug release in different dissolution media from IPN beads prepared using different polymer concentrations and gelling for $2 \mathrm{~h}$ in $2 \% \mathrm{w} / \mathrm{v} \mathrm{AlCl}_{3}$ solution

\begin{tabular}{ccccc}
\hline \multirow{2}{*}{$\begin{array}{c}\text { SCMX\%: } \\
\text { SAL\% }\end{array}$} & In acidic solution & \multicolumn{3}{c}{ In phosphate buffer solution } \\
\cline { 2 - 5 } & $\begin{array}{c}\mathrm{AUC}(\% \mathrm{mg} \cdot \mathrm{min} / \mathrm{ml}) \\
(\mathrm{Mean} \pm \mathrm{SD}, \mathrm{n}=4)\end{array}$ & $\begin{array}{c}\mathrm{t}_{50 \%}(\mathrm{~min}) \\
(\mathrm{Mean} \pm \mathrm{SD}, \mathrm{n}=4)\end{array}$ & $\begin{array}{c}\mathrm{t}_{80 \%}(\mathrm{~min}) \\
(\mathrm{Mean} \pm \mathrm{SD}, \mathrm{n}=4)\end{array}$ & $\begin{array}{c}\mathrm{AUC}(\% \mathrm{mg} \cdot \mathrm{min} / \mathrm{ml}) \\
(\mathrm{Mean} \pm \mathrm{SD}, \mathrm{n}=4)\end{array}$ \\
\hline $1: 1$ & $885.6 \pm 56.31$ & $94.8 \pm 5.20$ & $143.5 \pm 15.21$ & $14336.1 \pm 25.63$ \\
$1.5: 1.5$ & $729.2 \pm 35.50$ & $108.5 \pm 8.75$ & $174.1 \pm 10.11$ & $12553.9 \pm 27.75$ \\
$2: 2$ & $574.5 \pm 46.21$ & $132.4 \pm 6.31$ & $209.6 \pm 8.65$ & $10564.5 \pm 14.02$ \\
\hline
\end{tabular}

matrix [12]. As a result, the rigidity of gel matrix increases and free volume of the matrix decreases [19]. This hinders easy transport of drug molecules through the matrix and reduces drug release from the matrix.

\subsubsection{Effect of Swelling of IPN Bead}

The release of a drug from a polymeric matrix is controlled by the swelling behaviour of the polymer. To study the effect of swelling of IPN beads on drug release, swelling ratio of beads was measured in terms of water uptake at selected time intervals and the results have been represented in Figure 7. While the swelling ratio of the IPN beads was very low in acidic solution, the same property increased considerably in PB solution ( $\mathrm{pH}$ 6.8). The main functional group present in both the polymers that undergoes cross-linking with $\mathrm{Al}^{3+}$ ions is $-\mathrm{COOH}$ group. In acidic solution, $-\mathrm{COOH}$ group remains protonated and exerts insignificant electrostatic repulsive force. As a result, the beads swell to very less extent. At higher $\mathrm{pH}$ value of $\mathrm{PB}$ solution, $-\mathrm{COOH}$ group undergoes ionization which exerts electrostatic repulsion between the ionized groups, and results in higher swelling. Moreover, upon ionization, the counter ion concentration inside the polymeric network increases, and an osmotic pressure difference exists between the internal and external solutions of the beads. The increased osmotic pressure is balanced by the swelling of the beads [20]. The higher the swelling of the polymers, the higher is the drug release from the IPN beads. Thus the slower release of IBP in acidic solution and faster release in $\mathrm{PB}$ solution are related to the swelling behaviour of IPN beads in the respective dissolution media. It was further observed that increase in total polymer concentration from 2 to $4 \% \mathrm{w} / \mathrm{v}$ decreased the swelling of IPN beads in both the media. At low polymer concentration, the polymeric network is loose with a greater hydrodynamic free volume which allows more of the liquid to be absorbed and produces higher swelling. This, in turn, facilitates transport of the drug molecule through the matrix and causes higher drug release [21]. On the other hand, at higher polymeric concentration, opposite phenomenon takes place resulting in slower release of drug.

\subsubsection{Effect of Concentration of $\mathrm{AlCl}_{3}$}

The effect of the concentration of the cross-linking agent $\left(\mathrm{AlCl}_{3}\right)$ on the release profiles of the drug was studied with IPN beads prepared using $3 \% \mathrm{w} / \mathrm{v}$ total polymer concentration and gelling for $0.5 \mathrm{~h}$ in $2-8 \% \mathrm{AlCl}_{3}$ solution. Figure 8 showed that as the concentration of $\mathrm{AlCl}_{3}$

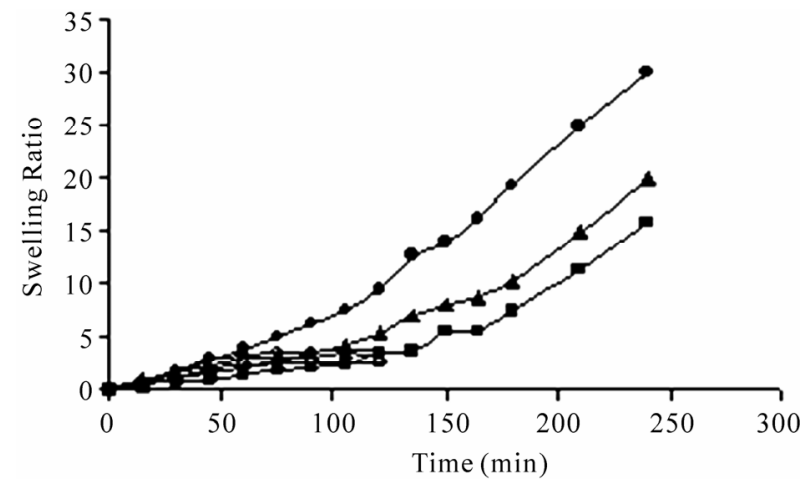

Figure 7. Swelling ratios of IPN beads, in acidic solution (open symbols) and phosphate buffer solution (closed symbols), prepared using different concentration of SCMX and SAL and gelling in $2 \% \mathrm{w} / \mathrm{v} \mathrm{AlCl}_{3}$ solution for $0.5 \mathrm{~h}$. Key: SCMX: SAL $=(\bigcirc)$ 1\%:1\%, $(\Delta)$ 1.5\%:1.5\%, ( $\square)$ 2\%:2\%

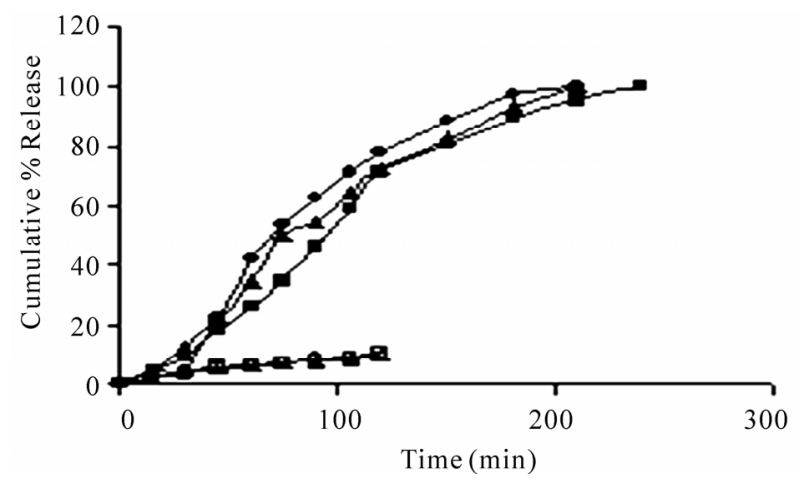

Figure 8. Release profiles of ibuprofen in acidic solution (open symbols) and phosphate buffer solution (closed symbols) from IPN beads prepared using SCMX: SAL $=1.5 \%$ : $1.5 \%$ and gelling for $0.5 \mathrm{~h}$ in different concentration of $\mathrm{AlCl}_{3}$ solution. Key: (O) $2 \%$ w/v, ( $\left.\Delta\right) 4 \% \mathrm{w} / \mathrm{v},(\square) 8 \% \mathrm{w} / \mathrm{v}$. Maximum SEM $=0.81(n=4)$ 
was increased during the preparation of beads, the release of drug increased in both the dissolution media. Statistical analysis in terms of ANOVA-1 also confirmed this phenomenon as the AUCs in acidic medium increased and $\mathrm{t}_{50 \%}, \mathrm{t}_{80 \%}$ decreased and AUCs increased in PB solution significantly. This unusual release behaviour could be explained in the following way. When the IPN beads were prepared with higher concentration of $\mathrm{AlCl}_{3}$, a thick outer gel layer might have been formed along the periphery of the beads. The thicker outer gel layer provided higher diffusional resistance to further influx of $\mathrm{Al}^{3+}$ ions resulting in the formation of inhomogeneous gel beads and less densely cross-linked matrix in the core of the beads. During dissolution study, once the outer thick gel layer swelled, quick drug release occurred from the beads. At lower concentration of $\mathrm{AlCl}_{3}, \mathrm{Al}^{3+}$ ions diffuse more uniformly into the beads and form homogenous gel beads resulting in slow drug release.

\subsubsection{Effect of Gelation Time}

The derived properties obtained from drug release profiles (Table 3) indicated that increase in gelation time decreased the drug release appreciably. The higher the gelation time, the greater is the cross-linking density and rigidity of the matrix which resulted in a fall in drug release.

\subsubsection{Effect of Drug Load}

The effect of drug load on the release dynamics of IBP was studied using IPN beads prepared using 3\% w/v total polymer concentration (SCMX:SAL in a weight ratio 1:1) and gelling for $0.5 \mathrm{~h}$ in $2 \% \mathrm{w} / \mathrm{v} \mathrm{AlCl}_{3}$ solution, and the results are shown in Figure 9. Increase in drug load from 20 to $60 \% \mathrm{w} / \mathrm{w}$ of total polymer decreased the drug release in both the dissolution media. Generally, higher drug load provides higher concentration gradient between the drug in the dosage form and the external dissolution medium and results in faster drug release. The release of a drug is governed not only by drug diffusion through the polymeric network but also by the relaxational process of the polymer on solvent penetration. Low drug load in IPN beads forms larger pore fraction resulting in higher swelling and consequently faster drug release. On the other hand, at higher drug load, larger crystalline domain of drug is formed in the beads. This causes reduction as well as shrinkage of pores of the matrix and results in fall in drug release. Decrease in drug release with increase in drug load from various IPN beads have been reported [5,18,22-23].

\subsubsection{Release Kinetics}

Drug release from a swellable matrix primarily depends on the degree of gelation, hydration, chain relaxation, and erosion of polymer. To understand the mode of drug transport through the IPN beads, the release data were fitted to the classical power law expression [24]

$$
\mathrm{M}_{\mathrm{t}} / \mathrm{M}_{\alpha}=\mathrm{Kt}^{\mathrm{n}}
$$

where $M_{t}$ and $M_{\alpha}$ are, respectively, the amount of drug released at time $\mathrm{t}$ and at infinite time, $\mathrm{K}$ represents a constant incorporating structural and geometrical characteristics of the dosage forms, $\mathrm{n}$ denotes the diffusion exponent indicative of the mechanism of drug release. Values of $n$ ranging from 0.45 to 0.5 indicate Fickian or diffusion controlled release, values of $\mathrm{n}$ ranging from 0.5 to 0.89 indicate non-Fickian or anomalous release, and values of $\mathrm{n}$ ranging from 0.89 to 1.0 indicate Case-II transport mechanism. By applying least squares method to release data, the values of $n$ were estimated and have been shown in Table $\mathbf{4}$ along with the correlation co-efficient $\left(\mathrm{r}^{2}\right)$. The results indicate that drug release in acidic medium followed non-Fickian mechanism and in PB solution drug release occured following case-II transport mechanism. When the swelling data of drug-free IPN beads were fitted to the above power law expression, it was found that swelling in acidic medium took place following the non-Fickian mechanism and that in PB solution followed Case-II transport mechanism (Table 4).

Table 3. Effect of gelation time on derived properties of drug release in different dissolution media from IPN beads prepared using different polymer concentration and gelling in $2 \% \mathrm{w} / \mathrm{v} \mathbf{A l C l}_{3}$ solution for different periods of time

\begin{tabular}{|c|c|c|c|c|c|c|c|c|}
\hline \multirow{3}{*}{$\begin{array}{c}\text { SCMX\%: } \\
\text { SAL\% }\end{array}$} & \multicolumn{2}{|c|}{ In acidic solution } & \multicolumn{6}{|c|}{ In phosphate buffer solution } \\
\hline & \multicolumn{2}{|c|}{ Gelation time $0.5 \mathrm{~h}$ Gelation time $2 \mathrm{~h}$} & \multicolumn{3}{|c|}{ Gelation time $0.5 \mathrm{~h}$} & \multicolumn{3}{|c|}{ Gelation time $2 \mathrm{~h}$} \\
\hline & $\begin{array}{c}\text { AUC } \\
(\% \mathrm{mg} \cdot \mathrm{min} / \mathrm{ml}) \\
(\mathrm{Mean} \pm \mathrm{SD} \\
\mathrm{n}=4)\end{array}$ & $\begin{array}{c}\text { AUC } \\
(\% \mathrm{mg} \cdot \mathrm{min} / \mathrm{ml}) \\
(\mathrm{Mean} \pm \mathrm{SD} \\
\mathrm{n}=4)\end{array}$ & $\begin{array}{c}\mathrm{t}_{50 \%}(\min ) \\
(\text { Mean } \pm \mathrm{SD} \\
\mathrm{n}=4)\end{array}$ & $\begin{array}{c}\mathrm{t}_{80 \%}(\min ) \\
(\mathrm{Mean} \pm \mathrm{SD} \\
\mathrm{n}=4)\end{array}$ & $\begin{array}{c}\text { AUC } \\
(\% \mathrm{mg} \cdot \mathrm{min} / \mathrm{ml}) \\
(\mathrm{Mean} \pm \mathrm{SD} \\
\mathrm{n}=4)\end{array}$ & $\begin{array}{c}\mathrm{t}_{50 \%}(\min ) \\
(\mathrm{Mean} \pm \mathrm{SD} \\
\mathrm{n}=4)\end{array}$ & $\begin{array}{c}\mathrm{t}_{80 \%}(\min ) \\
(\mathrm{Mean} \pm \mathrm{SD} \\
\mathrm{n}=4)\end{array}$ & $\begin{array}{c}\text { AUC } \\
(\% \mathrm{mg} \cdot \mathrm{min} / \mathrm{ml}) \\
(\mathrm{Mean} \pm \mathrm{SD}, \\
\mathrm{n}=4)\end{array}$ \\
\hline $1 \%: 1 \%$ & $970.1 \pm 22.63$ & $885.4 \pm 56.31$ & $77.5 \pm 7.86$ & $111.1 \pm 11.53$ & $13151.5 \pm 31.46$ & $94.8 \pm 5.20$ & $143.5 \pm 15.21$ & $14336.1 \pm 25.63$ \\
\hline $1.5 \%: 1.5 \%$ & $702.2 \pm 27.15$ & $729.2 \pm 35.50$ & $94.3 \pm 10.45$ & $148.1 \pm 9.45$ & $11117.7 \pm 20.46$ & $108.5 \pm 8.75$ & $174.1 \pm 10.11$ & $12553.9 \pm 27.75$ \\
\hline $2 \%: 2 \%$ & $565.5 \pm 13.36$ & $574.5 \pm 46.21$ & $119.7 \pm 12.61$ & $176.4 \pm 14.63$ & $9230.4 \pm 26.81$ & $132.4 \pm 6.31$ & $209.6 \pm 8.65$ & $10564.5 \pm 14.02$ \\
\hline
\end{tabular}


Table 4. Kinetic data (n) and correlation coefficient $\left(r^{2}\right)$ of (A) drug release and (B) swelling of IPN beads prepared by gelling in $2 \% \mathrm{w} / \mathrm{v} \mathrm{AlCl}_{3}$ solution for $0.5 \mathrm{~h}$

\begin{tabular}{|c|c|c|c|c|c|}
\hline \multirow{2}{*}{\multicolumn{2}{|c|}{ SCMX:SAL }} & \multicolumn{2}{|c|}{ In acidic solution } & \multicolumn{2}{|c|}{ In phosphate buffer solution } \\
\hline & & $n$ & $r^{2}$ & $\mathrm{n}$ & $r^{2}$ \\
\hline \multirow{3}{*}{ A } & $1 \%: 1 \%$ & 0.73 & 0.997 & 1.26 & 0.989 \\
\hline & $1.5 \%: 1.5 \%$ & 0.69 & 0.996 & 1.28 & 0.994 \\
\hline & $2 \%: 2 \%$ & 0.87 & 0.993 & 1.30 & 0.989 \\
\hline \multirow{3}{*}{ B } & $1 \%: 1 \%$ & 0.69 & 0.877 & 1.40 & 0.996 \\
\hline & $1.5 \%: 1.5 \%$ & 0.64 & 0.935 & 1.33 & 0.982 \\
\hline & $2 \%: 2 \%$ & 0.80 & 0.907 & 1.23 & 0.923 \\
\hline
\end{tabular}

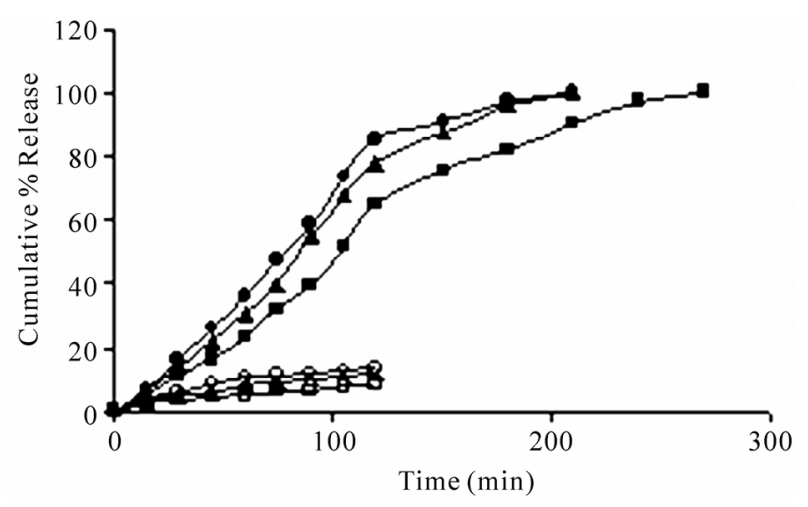

Figure 9. Effect of drug load on release of ibuprofen in acidic solution (open symbols) and phosphate buffer solution (closed symbols) from IPN beads prepared using SCMX:SAL $=1.5 \%: 1.5 \%$ and gelling for $0.5 \mathrm{~h}$ in $2 \% \mathrm{AlCl}_{3}$ solution. Key: drug load, (O) $20 \%$ w/v, ( $\Delta) 40 \%$ w/v, ( $\square$ ) $60 \% \mathrm{w} / \mathrm{v}$ of total polymer. Maximum $\mathrm{SEM}=1.27(\mathrm{n}=4)$

\section{Conclusions}

SCMX-SAL interpenetrating network beads were prepared by inotropic gelation method using $\mathrm{Al}^{3+}$ ions as cross-linking agent for both the polymers. Formation of IPN structure was verified by FTIR analysis and the absence of drug-polymer interaction in IPN beads was confirmed by FTIR, XRD, and DSC analysises. DEE of IPN beads were found to be reasonably high (91.15 to $99.50 \%$ ) and was not affected by formulation variables except the gelation time, the increase of which tended to decrease DEE. While the release of IBP decreased in both acidic (pH 1.2) and PB solution ( $\mathrm{pH}$ 6.8) with increase in total polymer concentration, gelation time, and drug load, the drug release increased in both the media with increase in the concentration of $\mathrm{AlCl}_{3}$. However, all the formulations showed considerably low release in acidic medium and the release followed non-Fickian transport mechanism due to poor swelling of the beads. Complete drug release was achieved in PB solution (pH 6.8) at different periods of time depending on the formulation variables and the release followed case II transport process due to swelling and erosion of the beads. The results of the study indicate that high drug-loaded IPN beads can be prepared using SCMX and SAL by ionotropic gelation process and could be used to minimize the release of IBP in acidic medium and to modulate the drug release in PB solution (pH 6.8).

\section{REFERENCES}

[1] M. Changez, K. Burugapalli, V. Koul and V. Chowdary, "The Effect of Composition of Poly (Acrylic Acid)-Gelatin Hydrogel on Gentamycin Sulphate Release in Vitro," Biomaterials, Vol. 24, No. 4, 2003, pp. 527-536.

[2] A. R. Kulkarni, K. M. Soppimath, T. M. Aminabhavi and W. E. Rudzinski, "In-Vitro Release Kinetics of Cefadroxyl Loaded Sodium Alginate Interpenetrating Network Beads," European Journal of Pharmaceutics and Biopharmaceutics, Vol. 51, No. 2, 2001, pp. 127-133.

[3] T. T. Hsieh, K. H. Hsieh, G. P. Simon and C. Tiu, "Interpenetrating Polymer Networks of 2-Hydroxylethyl Methacrylate Terminated Polyurethanes and Urethanes," Polymer, Vol. 40, No. 11, 1999, pp. 3153-3163.

[4] A. K. Bajpai, J. Bajpai and S. Shukla, "Water Sorption through Semi-Interpenetrating Polymer Network with Hydrophilic and Hydrophobic Chains," Reactive and Functional Polymers, Vol. 50, No. 1, 2002, pp. 9-21.

[5] A. Roy, A. K. Bajpai and J. Bajpai, "Designing Swellable Beads of Alginate and Gelatin for Controlled Release of Pesticide (Cypermethrin)," Journal of Macromolecular Science, Part A, Vol. 46, No. 9, 2009, pp. 847-859.

[6] P. Aslani and R. A. Kennedy, "Studies on Diffusion in Alginate Gels 1. Effect of Cross-Linking with Calcium or Zinc Ions on Diffusion of Acetaminophen," Journal of Controlled Release, Vol. 42, No. 1, 1996, pp. 75-82.

[7] T. L. Bowersock, H. HogenEsch, M. Suckow, P. Guimond, S.Martin, D. Borie, S.Torregrosa, H. Park and K.Park, "Oral Vaccination of Animals with Antigens Encapsulated in Alginate Microspheres," Vaccine, Vol. 17, No. 13-14, 1999, pp. 1804-1811.

[8] M. L. Gonzalez-Rodriguez, M. A. Holgado, C. Sanchez-Lafuente, A. M. Rabasco and A. Finni, "Alginate/ Chitosan Particulate Systems for Sodium Diclofenac Release," International Journal of Pharmaceutics, Vol. 232, No. 1-2, 2002, pp. 225-234.

[9] A. Halder, S. Maiti and B. Sa, "Entrapment Efficiency and Release Characteristics of Polyethyleneimine-Treated or Untreated Calcium Alginate Beads Loaded with Propranolol," International Journal of Pharmaceutics, Vol. 302, No. 1-2, 2005, pp. 84-94.

[10] N. P. Desai, A. Sojomihardjo, Z. Yao, N. Ron and P. Soon-Shiong, "Interpenetrating Polymer Networks of Alginate and Polyethylene Glycol for Encapsulation of Is- 
lets of Langerhans," Journal of Microencapsulation, Vol. 17, No. 6, 2000, pp. 677-690.

[11] S. G. Kumber and T. M. Aminabhavi, "Preparation and Characterisation of Interpenetrating Network Beads of Poly (Vinyl Alcohol)-Grafted-Poly (Acrylamide) with Sodium Alginate and their Controlled Release Characteristics of Cypermethrin Pesticides," Journal of Applied Polymer Science, Vol. 84, No. 3, 2002, pp. 552-560.

[12] Y. H. Liu, H.-F. Lian, C.-K. Chung, M.-C. Chen and H. -W. Sung, "Physically Cross-Linked Alginate/N,O-Carboxymethyl Chitosan Hydrogels with Calcium for Oral Delivery of Protein Drugs," Biomaterials, Vol. 26, No. 14, 2005, pp. 2105-2213.

[13] B. Sa and M. Setty, "Novel Gel Microbeads Based on Natural Polysaccharides," Indian Patent, No. 224992, 31 October 2008.

[14] S. Maiti, S. Roy, B. Mondal, S. Sarkar and B. Sa, "Carboxymethyl Xanthan Microparticles as a Carrier for Protein Delivery," Journal of Microencapsulation, Vol. 24, No. 8, 2007, pp. 743-756.

[15] S. Ray, S. Maiti and B. Sa, "Preliminary Investigation on the Development of Diltiazem Resin Complex Loaded Carboxymethyl Xanthan Beads," AAPS PharmSciTech, Vol. 9, No. 1, 2008, pp. 295-301.

[16] C. M. Setty, S. S. Sahoo and B. Sa, "Alginate-Coated Alginate-Polyethyleneimine Beads for Prolonged Release of Furosemide in Simulated Intestinal Fluid," Drug Development and Industrial Pharmacy, Vol. 31, No. 4-5, 2005, pp. 435-446.

[17] P. Piyakulawat, N. Praphairaksit, N. Chantarasiri and N. Muangsin, "Preparation and Evaluation of Chitosan/CarRageenan Beads for Controlled Release of Sodium Diclofenac," AAPS PharmSciTech, Vol. 8, No. 4, 2007, pp. $1-10$.

[18] A. P. Rokhade, S. A. Agnihotri, S. A. Patil, N. N. Mal- likarjuna, P. V. Kulkarni and T. M. Aminabhavi, "SemiInterpenetrating Polymer Network Microspheres of Gelatin and Sodium Carboxymethyl Cellulose for Controlled Release of Ketorolac Tromethamine," Carbohydrate Polymers, Vol. 65, No. 3, 2006, pp. 243-252.

[19] S. A. Agnihotri and T. M. Aminabhavi, "Development of Novel Interpenetrating Network Gellan Gum-Poly (Vinyl Alcohol) Hydrogel Microspheres for the Controlled Release of Carvedilol," Drug Development and Industrial Pharmacy, Vol. 31, No. 6, 2005, pp. 491-503.

[20] K. S. Soppimath, A. R. Kulkarni and T. M. Aminabhavi, "Chemically Modified Polyacrylamide-G-Guar Gum Based Crosslinked Anionic Microgels as PH Sensitive Drug Delivery Systems: Preparation and Characterization," Journal of Controlled Release, Vol. 75, No. 3, 2001, pp. 331-345.

[21] R. V. Kulkarni and B. Sa, "Novel PH-Sensitive Interpenetrating Network Hydrogel Beads of Carboxymethyl Cellulose-(Polyacryl Amide-Grafted-Alginate) for Controlled Release of Ibuprofen: Preparation and Characterization," Current Drug Delivery, Vol. 5, No. 4, 2008, pp. 256-264.

[22] S. Benita, A. Barkai and Y. U. Pathak, "Effect of Drug Loading Extent on the in Vitro Release Kinetic Behaviour of Nifedipine from Polyacrylate Microspheres," Journal of Controlled Release, Vol. 12, No. 3, 1990, pp. 213-222.

[23] K. S. Soppimath, A. R. Kulkarni and T. M. Aminabhavi, "Controlled Release of Antihypertensive Drug from the Interpenetrating Network Poly (Vinyl Alcohol)-Guar Gum Hydrogel Microspheres," Journal of Biomaterials Science Polymer Edition, Vol. 11, No. 1, 2000, pp. 27-43.

[24] P. L. Ritger and N. A. Peppas, "A Simple Equation for Description of Solute Release. II Fickian and Anomalous Release from Swellable Devices," Journal of Controlled Release, Vol. 5, No. 1, 1987, pp. 37-42. 